\title{
Judicious use of frozen section in parathyroid surgery
}

\author{
Serozsha A.S. Goonewardena \\ Nawaloka Hospital, Colombo
}

I read with great interest the article by Seneviratne and Gamage [1]. We should set the record straight with regard to the history of parathyroid (PT) surgery. Dr Felix Mandl performed the first parathyroidectomy for primary hyperparathyroidism (PHPT) on July 30, 1925, in Vienna on Herr Albert Jahne, a 38-year-old man with incapacitating osteitis fibrosa cystica [2]. This was a bilateral neck exploration (BNE). I have referred several PHPT patients with bilateral renal stones to reputed surgeons for PT surgery with eventual operative failure in most cases. In this context, the authors should be highly praised for the excellent results achieved. However, I beg to differ with the authors on a few salient facts [1].

(1) Title of the paper stating ' ..... in a resource poor setting' is very misleading. The authors worked in a modern health facility with facilities for neck ultrasonography and 99m Tc sestamibi scanning for preoperative localization, and frozen section (FS) and PTH assay for intraoperative assessment. In Sri Lanka, even if preoperative localization studies are negative, a common occurrence, BNE (a 4-gland exploration) is performed for PHPT. Although not stated, I assume that nearly all patients in the present series had had BNE. Preoperative localization studies are not necessary when planning a BNE [2,3].

(2) Intraoperative PTH (IOPTH) assay determines the completeness of removal of hyperfunctioning PT gland(s). Therefore, a postoperative PTH (15 min after surgery) in lieu of IOPTH is superfluous. Acceptable turnaround time for IOPTH is $20-30 \mathrm{~min}$.

(3) The authors have documented without elaborating, instances of incorrect gross identification of PT tissue by the surgeon. They should state the frequency of non-PT tissue removal associated with the gross examination (GE): PT misidentification rate, and how many were a lymph node, fat etc. If the details of GE in PT adenoma was stated, such as mahogany red-brown tissue / yellow-tan tissue, soft consistency, "fleshy" on cut surface etc. it would be more

Correspondence: Dr Serozsha A.S. Goonewardena

E-mail: drsasgurol@yahoo.com

Received: 30-05-2019 Accepted: 24-07-2019

(iD) http://orcid.org/0000-0002-7353-3356

DOI: http://doi.org/10.4038/sljs.v37i2.8632

The Sri Lanka Journal of Surgery 2019; 37(2): 32- 33 credible and scientific.

(4) Rather than stating that patients were followed up during the last 5 years with no evidence of recurrence, when the last patient was treated in December 2018 (a 4-month followup), it would be meaningful to state the mean / median followup in months. Recurrent hyperparathyroidism occurs after a few years.

(5) Dewan et al.concluded that FS is not recommended for typical PT appearance: large $(>1 \mathrm{~cm})$, tan-mahogany, soft, "fleshy" on the cut surface. They emphasize the situations that warrant the judicious use of FS: inexperienced PT surgeon, multiple gland disease, ectopic gland location, technically difficult primary surgeries and revision surgery [4].

(6) The authors fail to explain why intraoperative FS during PT surgery became obsolete in high-volume centres. Parathyroid surgeons should do their ultrasonography (surgeon-performed) if focused parathyroidectomy is their ultimate goal since $85-88 \%$ of cases are single PT adenoma and until then do FS selectively.

\section{References}

1. Seneviratne BU, Gamage B. Role of routine frozen sections for parathyroid exploration in a resource poor setting. Sri Lanka Journal of Surgery 2019; 37: 16-21.

https://doi.org/10.4038/sljs.v37i1.8599

2. Elaraj D, Sturgeon C. Operative treatment of primary hyperthyroidism: Balancing cost-effectiveness with successful outcomes. Surgical Clinics of North America 2014; 94: 607-623. https://doi.org/10.1016/j.suc.2014.02.011

3. McGill J, Sturgeon C, Kaplan SP et al. How does the operative strategy for primary hyperparathyroidism impact the findings and cure rate ? A comparison of 800 parathyroidectomies. Journal of American College of Surgeons 2008; 207 (2): 246-249

https://doi.org/10.1016/j.jamcollsurg.2008.01.066

4. Dewan AK, Kapadia SB, Hollenbeck CS et al. Is routine frozen section necessary for parathyroid surgery? Otolarygology and Head and Neck Surgery 2005; 133 (6): 857-862

https://doi.org/10.1016/j.otohns.2005.05.001 


\section{The Authors' Reply:}

\section{3/07/2019 \\ Editor, \\ The Sri Lanka Journal of Surgery, College of Surgeons of Sri Lanka}

\section{Dear Editor,}

Thank you for forwarding the comments of the scientific article on "Role of routine frozen sections for parathyroid exploration in a resource poor setting" co-authored by Bimalka Seneviratne and Bawantha Gamage. This was an inspiring topic that motivated us to compile our data and disseminate the scientific findings.

We are pleased to submit the following clarifications;

(01) It is interesting to note that we have found intraoperative frozen sections as a valuable guide for parathyroid exploration. Novel imaging studies are far too costly, nonfunctional time to time due to technical issues and unavailable without prior notice due to the shortage of human resources. Intraoperative frozen section is cost effective, accurate and can be easily arranged within a short period of time.

The correspondent has mentioned that he/she has referred several patients with primary hyperparathyroidism (PHPT) for reputed surgeons for surgery but there were operative failures in most cases. We assume this is mostly due to the inaccurate identification of parathyroid adenoma. Despite preoperative localization by imaging modalities, we have also got several patients referred to us for re-explorations. We are in the opinion that this problem could have been easily avoided by intraoperative frozen section (FS) by an experienced pathologist. Our main objective is to highlight this point, as FS can be done in almost all laboratories where consultant surgeons and pathologist are working even though those centres are lacking sophisticated facilities like Sestamibi scan facility or intraoperative PTH assays. Although the study was done in a center with all those facilities, we have shown that FS is to confirm the accurate removal of Parathyroid adenoma. Therefore, in resource poor settings FS can be used to avoid reoperations.

We did not do bilateral neck exploration (BNE) in our 22 patients with parathyroid adenoma. They all had local exploration of the affected gland. All patients with adenoma had preoperative USS neck a few hours prior to surgery and out of them adenoma was identified in 20 patients. On those patients, skin overlying the corresponding adenoma was marked with the document-ation of the depth of the tumour from the skin.
(02) In the current study frozen section diagnosis was complemented by post-operative parathyroid levels. There was a statistically significant association with the intraoperative diagnosis and post-operative parathyroid hormone level.

We have mentioned in the discussion that we took blood samples for parathhormone levels 15 mintues after successful parathyroid removal. This was done during the surgery and we agree the terminology should have been post parathyroid removal or intraoperative rather than post-operative.

(03) Gross examination findings of surgical specimens are given in table (01). Sensitivity and specificity were highly satisfactory and the concordance between the surgeon and pathologist was $>95 \%$. Non-parathyroid tissue which included small lymph nodes, fat globules and thyroid nodules are mentioned in table $(02)$.

We did not elaborate the instances of incorrect gross identification as once this information was conveyed to the surgeon during the surgery further exploration was carried out and adenoma was accurately identified and sent for FS in all instances.

(04) Follow up of patients up to date has shown no evidence of disease recurrence. We agree that follow up would have been given as mean or median in months in our cohort of patients.

In view of the small sample size the mean was not calculated.

(05) Gross examination may not be $100 \%$ reliable. In the fresh state thyroid tissue could mimic parathyroid, thus causing confusion.

Yes, we do, agree in certain instances parathyroid can be confirmed by the naked eye appearance.

Authors did not want to carry the slightest risk for a second surgery, hence the specimens were subjected for frozen section diagnosis.

(06) In contrast to developed countries which have readily available sophisticated imaging techniques, in resource poor settings intraoperative frozen sections will continue to play a pivotal role during parathyroid exploration.

Authors do not feel FS is obsolete in resource poor settings and it will definitely minimize reoperation rates.

\section{Co-authors: \\ Prof. Bimalka Seneviratne \\ Dr. Bawantha Gamage}

23/07/2019 